\title{
Nitazoxanide with hydroxychloroquine in patients with enhanced risk for severe COVID-19 infection
}

SARS-CoV-2 disease pathogenesis is multidimensional owing to both direct viral infection and host $\mathrm{CD} 8^{+} \mathrm{T}$-cell immune response. ${ }^{[1]}$ In SARS, cell-mediated immunity, especially $\mathrm{CD}^{+} \mathrm{T}$-cell response, is responsible for host damage, including acute respiratory distress syndrome (ARDS) and multiorgan failure. ${ }^{[1]}$ Designing drug therapies based on viral biology and host immune responses, as well as the interplay between them, will enable a targeted approach against SARS-CoV-2 infection. An ideal therapy regimen should (i) target and reduce viral replication; (ii) upregulate host innate immune antiviral responses; and (iii) downregulate the virus-induced immune dysregulation that leads to lung damage and cytokine storm involved in the pathogenesis of ARDS and multiorgan failure. ${ }^{[1]}$

Nitazoxanide (NTZ) is an antiprotozoal drug that is Food and Drug Administration-approved for treating Cryptosporidium and Giardia, and has an excellent safety record for variety of indications. ${ }^{[2]}$ It has demonstrated and proven broad antiviral activity. Indeed, NTZ amplifies cytoplasmic RNA sensing and augments type I IFN antiviral responses. It has been shown to have antiviral activity against several viruses including Ebola, hepatitis B and C, rotavirus and norovirus. ${ }^{[3]}$ With regard to respiratory viral infections, NTZ was evaluated in uncomplicated influenza and demonstrated a reduction in median time to symptom recovery. ${ }^{[4]}$ By contrast, it failed to show benefit in hospitalised patients with severe influenza, suggesting that, as with oseltamivir (Tamiflu), it likely needs to be given early in the course of the disease. ${ }^{[5]}$ NTZ has also demonstrated in vitro activity against SARS-CoV-2. ${ }^{[6]}$

Hydroxychloroquine, a drug with a good global safety profile, has been shown in several studies to have antiviral properties (it prevents the virus binding to human epithelial cells and interrupts viral replication). It has direct SARS-CoV-2 antiviral effects in in vitro studies, and in early clinical trials has been associated with a reduction in viral load. Several clinical trials are ongoing, but almost all of these studies are or have investigated hydroxychloroquine late in the course of COVID-19 (i.e. in patients who have been hospitalised or who are already receiving respiratory support). Perhaps if hydroxychloroquine is given early in the disease, or in combination with other antiviral drugs such as NTZ, it might prove to be effective. Padmanabhan ${ }^{[1]}$ proposes that combining hydroxychloroquine and NTZ for treatment of COVID-19 is likely to have synergistic activity against SARS$\mathrm{CoV}-2$, acting against distinct pathways. Hydroxychloroquine reduced viral replication, reduced viral entry (by interfering with ACE2 glycosylation) and has sustained immunomodulatory properties, given its long half-life. However, there is no effect on interferon production, which is inhibited by SARS-CoV-2. NTZ upregulates the interferon innate immune pathway, thus disturbing viral replication.

Thus the proposed treatment regimen with hydroxychloroquine and NTZ will (i) diminish the severity of illness by reducing viral titers; and (ii) rescue the innate immune system dysregulation brought about by the viral infection. This will reduce the high mortality in the older vulnerable population, thereby allowing our healthcare system a more controlled disease response.

\section{Phindile Gina}

Centre for Lung Infection and Immunity, Division of Pulmonology, Department of Medicine and UCT Lung Institute, University of Cape Town, South Africa

1. Padmanabhan S. Potential dual therapeutic approach against SARS-CoV-2/COVID-19 with nitazoxanide and hydroxychloroquine. ResearchGate March 2020; (epub ahead of print). https://doi.org.10.13140/RG.2.2.28124.74882

2. Rossignol JF, La Frazia S, Chiappa L, Ciucci A, Santoro MG. Thiazolides, a new class of anti-influenza molecules targeting viral hemagglutinin at the post-translational level. J Biol Chem 2009;284(43):29798-29808. https://doi.org/10.1074/jbc.m109.029470

3. Jasenosky LD, Cadena C, Mire CE, et al. The FDA-approved oral drug nitazoxanide amplifies host antiviral responses and inhibits Ebola virus. iScience 2019;19:12791290. https://doi.org/10.1016/j.isci.2019.07.003

4. Haffizulla J, Hartman A, Hoppers M, et al. Effect of nitazoxanide in adults and adolescents with acute uncomplicated influenza: A double-blind, randomised, placebo-controlled, phase 2b/3 trial. Lancet Infect Dis 2014;14(7):609-618. https:// doi.org/10.1016/s1473-3099(14)70717-0

5. Gamino-Arroyo AE, Guerrero ML, McCarthy S, et al. Efficacy and safety of nitazoxanide in addition to standard of care for the treatment of severe acute respiratory illness. Clin Infect Dis 2019;69(11):1903-1911. https://doi.org/10.1093/ $\mathrm{cid} / \mathrm{ciz} 100$

6. Wang M, Cao R, Zhang L, et al. Remdesivir and chloroquine effectively inhibit the recently emerged novel coronavirus (2019-nCoV) in vitro. Cell Res 2020;30(3):269271. https://doi.org/10.1038/s41422-020-0282-0

7. Korba BE, Montero AB, Farrar K, et al. Nitazoxanide, tizoxanide and other thiazolides are potent inhibitors of hepatitis $\mathrm{B}$ virus and hepatitis $\mathrm{C}$ virus replication. Antiviral Res 2008;77(1):56-63. https://doi.org/10.1016/j.antiviral.2007.08.005 